\title{
A PROPERTY OF THE LEVEL LINES OF A REGION WITH A RECTIFIABLE BOUNDARY*
}

\author{
BY G. A. PFEIFFER
}

1. Introduction. Before stating the result of this paper let me recall that a level line of a region $\dagger$ in a plane is the locus of the equation $g(x, y ; a, b)=c$, where $g(x, y ; a, b)$ is the Green's function of the region which has the point $(a, b)$ as its pole, and $c$ is a positive constant. The set of all level lines of the region, with the point $(a, b)$ fixed and $c$ any positive constant, is called a pencil of level lines of the region, and the fixed point $(a, b)$ is called the pole of that pencil. Any pencil $\Sigma$ of level lines of a region which is in a plane and which has a connected boundary containing more than one point, is the image of the set of circles concentric with and interior to any circle $K$ under any transformation $\Pi$ which maps in a one-to-one and conformal way the interior of $K$ on $\Sigma$, such that the pole of the pencil of level lines corresponds to the center of $K$; and, conversely, the image of the set of circles concentric with and interior to a circle $K$ under any transformation $\Pi$ which maps in a one-to-one and conformal way the interior of $K$ on a planar region $\Sigma$ is a pencil of level lines of $\Sigma$, which has the image under $\Pi$ of the center of $K$ as its pole. Thus, a pencil of level lines of $\Sigma$ is a one-parameter set of simple closed curves, and the value of the parameter $t$ of a level line $G$ of such a pencil of level lines may be taken as the length of the radius of the circle to which $G$ corresponds under $\Pi$. Taking $K$ as the unit circle, then $t$ varies between 0 and 1 . The symbol $\left[G_{t}\right]$ denotes a pencil of level lines of the region

\footnotetext{
* Presented to the Society, May 7, 1927, as part of a paper of the title Certain sequences of curves which approach a rectifiable boundary from within.

$\dagger$ A region in a plane is a set of points in a plane such that there exists a planar neighborhood of each point of the set which contains only points of the set.
} 
$\Sigma$ such that the level line $G_{t}$ corresponds under $\Pi$ to the circle which is concentric with the unit circle and which has a radius of length $t(0<t<1)$.

Now, if $\Lambda(t)$ denotes the function of $t$ defined for $0<t<1$ and such that $\Lambda\left(t_{1}\right)$ is the length of the level line $G_{t_{1}}$ of the pencil of level lines $\left[G_{t}\right], 0<t<1$, then it is a result of a theorem of Hardy, referred to below, that $\Lambda(t)$ is an increasing continuous function of $t$ for $0<t<1$; that is, if $0<t_{1}<t_{2}<1$, then $\Lambda\left(t_{1}\right)<\Lambda\left(t_{2}\right)$. It is a simple consequence of the formula for the length of an analytic transform of a rectifiable curve, which is derived in $\$ 2$ below, that $\lim _{t \rightarrow 0} \Lambda(t)=0$ and nothing further is said about that; but much of the following proof is devoted to showing that if the boundary of $\Sigma$ is a rectifiable simple closed curve, then $\lim _{t \rightarrow 1} \Lambda(t)$ is the length of the boundary of $\Sigma$.

The result of this paper is contained in the following two theorems. The theorems are closely connected and their proofs are combined in the demonstration which follows.

THEOREM. The function $\Lambda(t)$ of $t$, which is defined in the interval $0 \leqq t \leqq 1$, and which is such that $\Lambda\left(t_{1}\right)$, if $0<t_{1}<1$, is the length of the level line $G_{t_{1}}$ of the pencil $\left[G_{t}\right], 0<t<1$, of level lines of the planar region $\Sigma$ whose boundary is a rectifiable simple closed curve and such that $\Lambda(0)=0$ and $\Lambda(1)=$ the length of the boundary of $\Sigma$, is an increasing* continuous function of $t$ in the (closed) interval $0 \leqq t \leqq 1$.

Definition. An approximating sequence of regions of the region $\Sigma$ is a sequence of regions $\left\{\Sigma_{n}\right\}, n=1,2,3, \cdots$, such that every limit point of each $\Sigma_{n}$ is a point of $\Sigma$ and every point of $\Sigma$ belongs to all but a finite number of the regions $\Sigma_{n} \dagger$

* That is, if $0 \leqq t_{1}<t_{2} \leqq 1$, then $\Lambda\left(t_{1}\right)<\Lambda\left(t_{2}\right)$.

$\dagger$ It follows readily from this definition that if the region $\Sigma$ is bounded then an approximating sequence of regions $\left\{\Sigma_{n}\right\}, n=1,2,3, \cdots$, of the region $\Sigma$ contains a subsequence of regions $\left\{\Sigma_{n_{i}}\right\}, i=1,2,3, \cdots$, which is such that (a) every limit point of any region $\Sigma_{n i}$ belongs to $\Sigma$ and to the succeeding region $\Sigma_{n_{i+1}}$ and (b) every point of $\Sigma$ is in all but a finite number of the regions $\Sigma_{n_{i}}$. 
Definition. An approximating sequence of curves of the region $\Sigma$ is a sequence of curves $\left\{C_{n}\right\}, n=1,2,3, \cdots$, such that each curve $C_{n}$ is the boundary of a region $\Sigma_{n}$ of an approximating sequence of regions $\left\{\Sigma_{n}\right\}, n=1,2,3, \cdots$, of the region $\Sigma$.

THEOREM. If the boundary of a planar region $\Sigma$ is a simple closed curve which is rectifiable, then there exist approximating sequences of curves $\left\{C_{n}\right\}, n=1,2,3, \cdots$, of the region $\Sigma$ such that the curves $C_{n}$ are level lines of any given pencil of level lines of $\Sigma$ and, if $l_{n}$ denotes the length of the curve $C_{n}$, $l_{n}<l_{n+1}$ and $\lim _{n \rightarrow \infty} l_{n}$ is the length of the boundary of $\Sigma$.

2. The Length of an Analytic Transform of a Rectifiable Curve. Let the function $w=f(z)$ be analytic in the interior, $i(K)$, of the unit circle, $K$, and map in a one-to-one way $i(K)$ on the region $\Sigma$ of the theorem. Let $C$ be a rectifiable curve in $i(K)$ and $C^{\prime}$ its image under the transformation $w=f(z)$. Then the length, $l^{\prime}$, of $C^{\prime}$ is

$$
\lim _{n \rightarrow \infty}\left(\left|\Delta w_{n_{1}}\right|+\left|\Delta w_{n_{2}}\right|+\cdots+\left|\Delta w_{n_{r}}\right|\right) \text {, }
$$

where $\Delta w_{n_{i}}=f\left(z_{n_{i}}\right)-f\left(z_{n_{i-1}}\right)$, where $z_{n_{0}}, z_{n_{1}}, z_{n_{2}}, \cdots, z_{n_{r}}$, $z_{n_{r+1}}=z_{0}$ are points on $C$ such that $\left|z_{n_{i}}-z_{n_{i-1}}\right|<\delta_{n}>0$, and where $\lim _{n \rightarrow \infty} \delta_{n}=0$.

If $\Delta s_{n_{i}}$ is the length of the arc of $C$ whose end points are $z_{n_{i}}$ and $z_{n_{i-1}}$ and which does not contain as an inner point the point $z=z_{n_{0}}$, then

$$
l^{\prime}=\lim _{n \rightarrow \infty} \sum_{i=1}^{r}\left|\frac{\Delta w_{n_{i}}}{\Delta z_{n_{i}}}\right| \cdot \Delta s_{n_{i}}, \quad \Delta z_{n_{i}}=z_{n_{i}}-z_{n_{i-1}} ;
$$

and, because of the uniformity of the approach of $\Delta w / \Delta z$ to $d w / d z$ along $C, d w / d z$ being continuous on $C$, it follows that

Hence

$$
l^{\prime}=\lim _{n \rightarrow \infty} \sum_{i=1}^{r}\left(\lim _{z \rightarrow z_{i}}\left|\frac{\Delta w}{\Delta z}\right|\right) \Delta s_{n_{i}} .
$$

$$
l^{\prime}=\int_{C}\left|\frac{d w}{d z}\right| d s .
$$


3. The Level Lines of a Polygonal Region. Let $i(J)$ denote a region whose boundary is a simple polygon $J$, and let $w=f(z)$ be a function which is analytic in the interior $i(K)$ of the unit circle $K$ and which maps in a one-to-one way $i(K)$ on $i(J)$. Then $w=f(z)$ is analytic at any point of the circle $K$ whose image is not a vertex of $J$ and if $w=f(a)$ is a vertex of $J$, then at any point of $i(K)$ different from $z=a$ in some neighborhood of $z=a$ the derivative of $w=f(z)$ is $(z-a)^{\mu} \lambda(z)$, where $\lambda(z)$ is analytic at $z=a$ and $-1<\mu<1$. $^{*}$ In fact, $\mu=\alpha / \pi-1$, where $\alpha$ is the measure in radians of the interior angle of the polygon $J$ whose vertex is the point $w=f(a)$.

Let the point $w=f\left(a_{i}\right)$ be a vertex of the polygon $J$ and $U_{\sigma_{i}}$ a neighborhood of $z=a_{i}$ such that at every point of $i(K)$ which is in $U_{a_{i}}$ and different from $z=a_{i}, f^{\prime}(z)=\left(z-a_{i}\right)^{\mu_{i}} \lambda_{i}(z)$, where $\lambda_{i}(z)$ is analytic at $z=a_{i}$ and $-1<\mu_{i}<1$. Further, let $\Gamma_{i}$ denote a circular arc concentric with $K$, and contained in $U_{a_{i}}$, such that its mid-point $z=b_{i}$ is on the radius of $K$ through $z=a_{i}$. Let $z=c_{i}$ be an end point of this arc. Then, if $-1<\mu_{i}<0$ and $M_{i}$ is a bound of $\left|\lambda_{i}(z)\right|$ in $U_{a_{i}}$,

$$
\int_{\Gamma}\left|f^{\prime}(z)\right| d s \leqq M_{i} \int_{\Gamma_{i}}\left(\left|z-a_{i}\right|\right)^{\mu i} d s \leqq M_{i} \int_{\Gamma_{i}}\left(\left|z-b_{i}\right|\right)^{\mu_{i}} d s .
$$

If $\eta$ is an arbitrary positive number, then there exists a positive number $\xi$ such that $\left|z-b_{i}\right| /\left(\overparen{z b}_{i}\right) \geqq 1-\eta$ if $z \bar{b}_{i}<\xi$, where $z \bar{b}_{i}$ denotes the length of the sub-arc of $\Gamma_{i}$ whose end points are $z=z$ and $z=b_{i}$. Then

$$
\begin{aligned}
& \int_{\Gamma_{i}}\left(\left|z-b_{i}\right|\right)^{\mu_{i}} d s \leqq(1-\eta)^{\mu_{i}} \int_{\Gamma_{i}}\left(\widehat{z b}_{i}\right)^{\mu_{i}} d s \\
= & 2(1-\eta)^{\mu_{i}} \int_{0}^{l_{i} / 2} s^{\mu_{i}} d s=2(1-\eta)^{\mu_{i}} \frac{1}{\mu_{i}+1} \cdot \frac{l^{\mu_{i}+1}}{2^{\mu_{i}+1}},
\end{aligned}
$$

* Bieberbach, Lehrbuch der Funktionentheorie, vol. II, p. 34 and p. 37. Also Study, Vorlesungen über ausgewählte Gegenstände der Geometrie, Part II, p. 85. 
where $l_{i}$ is the length of $\Gamma_{i}$ and if $\eta<1 / 2$, then

$$
\int_{\Gamma_{i}}\left(\left|z-b_{i}\right|\right)^{\mu_{i}} d s<\frac{1}{2^{\mu_{i}}\left(\mu_{i}+1\right)} l_{i}^{\mu_{i+r}} .
$$

Again, if $0 \leqq \mu_{i}<1$ and $M_{i}$ is a bound of $\left|\lambda_{i}(z)\right|$ in $U_{a_{i}}$, we have

$$
\begin{aligned}
\int_{\Gamma_{i}}\left|f^{\prime}(z)\right| d s & \leqq M_{i} \int_{\Gamma_{i}}\left(\left|z-a_{i}\right|\right)^{\mu_{i}} d s \leqq M_{i} \int_{\Gamma_{i}}\left|c_{i}-a_{i}\right| d s \\
& \leqq M_{i}\left(\left|a_{i}-b_{i}\right|+\frac{l_{i}}{2}\right) l_{i},
\end{aligned}
$$

where $l_{i}$ is the length of the arc $\Gamma_{i}$. Hence if $\epsilon$ is any positive number there exists a positive number $\delta_{i}$ such that if $\Gamma_{i}$ is any circular arc which is concentric with and either interior to or on the given circle $K$ and contained in $U_{a_{i}}$ and which has a length $l_{i}<\delta_{i}$, then

$$
\int_{\Gamma_{i}}\left|f^{\prime}(z)\right| d s<\epsilon
$$

Now, let $\sigma_{i}$ denote an arc on the circle $K$ which has $z=a_{i}$ as its mid-point and a length $l_{i}$ which is less than $\delta_{i}$ and, further, such that no two $\operatorname{arcs} \sigma_{i}$ have a point in common and let $R$ denote the set of all points which are interior to $K$ and which do not belong to any sector bounded by the arc $\sigma_{i}$ and the radii of $K$ through its end points; also let $\bar{R}$ denote the set of points consisting of the points of $R$ and of the boundary of $R$. Then $w=f^{\prime}(z)$ is analytic in $\bar{R}$ and hence there exists a positive number $\delta$ such that $\left|f^{\prime}\left(z_{1}\right)-f^{\prime}\left(z_{2}\right)\right|<\epsilon$ if $z=z_{1}$ and $z=z_{2}$ are any two points in $\bar{R}$ such that $\left|z_{1}-z_{2}\right|<\delta$. Now, if $d$ is a positive number less than the radius of each $U_{a_{i}}$, let $K^{\prime}$ denote a circle interior to and concentric with $K$ and having a radius which differs from that of $K$ by less than $d$ and also less than $\delta$. Then let $\tau_{i}$ denote any arc of $K$ which contains no $\operatorname{arc} \sigma_{i}$ and whose end points are also end points of $\operatorname{arcs} \sigma_{i}$ and let $\sigma_{i}{ }^{\prime}$ and $\tau_{i}^{\prime}$ denote the arcs of $K^{\prime}$ which are composed of the points of intersection of the circle $K^{\prime}$ 
and the radii of $K$ through all the points of $\sigma_{i}$ and all the points of $\tau_{i}$ respectively. It follows that

$$
\int_{\tau_{i^{\prime}}}\left|f^{\prime}(z)\right| d s=\int_{\tau_{i}} \frac{r-d}{r}\left(\left|f^{\prime}(z)\right|+\eta(z)\right) d s,
$$

where $r$ is the radius of $K$ and $|\eta(z)|<\epsilon$. If $h$ denotes the length of the polygon $J$ and $h^{\prime}$ the length of the transform of $K^{\prime}$ under the transformation $w=f(z)$ and $n$ the number of vertices of $J$, then

$$
\begin{aligned}
h^{\prime}=\sum_{i=1}^{n} \int_{\sigma_{i^{\prime}}}\left|f^{\prime}(z)\right| d s+\sum_{i=1}^{n} \int_{\tau_{i^{\prime}}}\left|f^{\prime}(z)\right| d s \\
<n \epsilon+\sum_{i=1}^{n} \int_{\tau_{i}}\left|f^{\prime}(z)\right| d s+\frac{r-d}{r} \epsilon h,
\end{aligned}
$$

and

$$
h^{\prime}>\sum_{i=1}^{n} \int_{\tau_{i}}\left|f^{\prime}(z)\right| d s-\frac{d}{r} h-\frac{r-d}{r} \epsilon h .
$$

Since

$$
h-n \epsilon<\sum_{i=1}^{n} \int_{\tau_{i}}\left|f^{\prime}(z)\right| d s<h,
$$

it follows that

$$
h-n \epsilon-\frac{d}{r} h-\frac{r-d}{r} \epsilon h<h^{\prime}<n \epsilon+h+\frac{r-d}{r} \epsilon h .
$$

Now if $d<\epsilon$, then

$$
h-\left(n+\frac{h}{r}+h\right) \epsilon<h^{\prime}<h+(n+h) \epsilon,
$$

or

$$
|h-| h^{\prime}<\left(n+\frac{h}{r}+h\right) \epsilon .
$$

Hence if $\rho$ is any positive number and $d$ is sufficiently small then $\left|h-h^{\prime}\right|<\rho$.

From this result follows immediately, as far as it concerns the $\lim _{n \rightarrow \infty} l_{n}$, the special case of the second theorem of the 
paper in which the region $\Sigma$ is the interior of a simple polygon in a plane.

4. The Region $\Sigma$ in General. Let $\Sigma$ be a region in the wplane and let the boundary of $\Sigma$ be a rectifiable simple closed curve, $C$. Then no level line of $\Sigma$ has a length greater than the length of the boundary of $\Sigma$. For let there exist a level line of $\Sigma$, say $G$, which has a length, $g$, which is greater than the length, $l$, of $C$. It is assumed that $w=0$ is an interior point of $G$; no loss of generality follows from this assumption. Then there exists a function $w=f(z)$ which is analytic in the unit circle and which maps in a one-to-one and conformal way the interior of the unit circle on $\Sigma$ such that $f(0)=0$ and $f^{\prime}(0)=1$ and such that $G$ is the image under the transformation $w=f(z)$ of a circle, $H$, concentric with the unit circle, $K$. Further, let $\left\{P_{n}\right\}, n=1,2,3, \cdots$, be a sequence of simple polygons inscribed in $C$ which are such that $\lim _{n \rightarrow \infty} d_{n}=0$, where $d_{n}$ is the length of a side of $P_{n}$ which is not shorter than any other side of $P_{n}$, and such that $w=0$ is an interior point of each $P_{n}$. Then there exists a function $w=f_{n}(z)$ which maps the interior of the circle $|z|=1$ on the interior of $P_{n}$ in a one-to-one and conformal way such that the point $w=0$ corresponds to the point $z=0$ and the derivative of the function $w=f_{n}(z)$ at $z=0$ is unity. By a theorem* of Caratheodory, and the fact that there is only one function which maps the interior of the unit circle on $\Sigma$ in a one-to-one and conformal way such that $w=0$ corresponds to $z=0$ and the derivative of the mapping function at $z=0$ is unity, it follows that the sequence of functions $\left\{w=f_{n}(z)\right\}, n=1,2$, $3, \cdots$, approaches the function $w=f(z),|z|<1$, uniformly on any closed set of points which is contained in the interior of the unit circle. Consequently the sequence of derivatives of the functions $w=f_{n}(z),\left\{w=f_{n}^{\prime}(z)\right\}, n=1,2,3, \cdots$, converges uniformly to the derivative of $w=f(z)$ on any closed set of points which is in the interior of the unit circle.

* See Mathematische Annalen, vol. 72 (1912), pp. 120-126. Also Bieberbach, Lehrbuch der Funktionentheorie, vol. II, pp. 12-15. 
Now, let $\nu$ be a positive number less than $g-l$ and $p$ a positive integer such that

for $z$ on $H$. Then

$$
\left|f^{\prime}(z)-f_{p}^{\prime}(z)\right|<\frac{\nu}{2 \pi}
$$

$$
\left|f_{p}^{\prime}(z)\right|=\left|f^{\prime}(z)\right|+\eta(z),
$$

where $|\eta(z)|<\nu / 2 \pi$ for $z$ on $H$, and

$$
\int_{H}\left|f_{p}^{\prime}(z)\right| d s=\int_{H}\left|f^{\prime}(z)\right| d s+\int_{H} \eta(z) d s .
$$

But

$$
\int_{H}\left|f^{\prime}(z)\right| d s=g \text { and } \int_{H}\left|f_{p}^{\prime}(z)\right| d s
$$

is the length of the image of $H$ under the transformation $w=f_{p}(z),|z|<1$. The latter image is a level line of the pencil of level lines of the polygonal region bounded by $P_{p}$, which has the point $w=0$ as its pole. If the length of this level line is denoted by $g_{p}$, then $g_{p}>g-\nu$ and hence $g_{p}>l$.

According to the result for polygonal regions which was obtained above, there exists a circle, $H^{\prime}$, with center $z=0$ and a radius of length less than unity but greater than the length of a radius of $H$ such that the length, $g_{p}^{\prime}$, of the image of $H^{\prime}$ under the transformation $w=f_{p}(z)$ differs from the length, $l_{p}$, of $P_{p}$ by an amount less than $g_{p}-l$. Since $l_{p}<l$ it follows that $g_{p}^{\prime}<g_{p}$. But this result contradicts the fact that by a theorem* of Hardy in connection with the formula for the length of an analytic transform of a rectifiable curve, which is given above. It follows that $g_{p}^{\prime}>g_{p}$.

* See Proceedings of the London Mathematical Society, (2), vol. 14 (1915), pp. 269-277. Also Landau, Ergebnisse der Funktionentheorie, p. 85. The theorem is that if $w=f(z)$ is analytic and not constant for $|z|<R$, then $\int_{0}^{2 \pi}\left|f\left(r e^{i \theta}\right)\right| d \theta, z=r e,{ }^{i \theta} 0 \leqq \theta \leqq 2 \pi$ and $0<r<R$, is a continuous increasing function of $r$ for $0<r<R$.

Only a special case of this theorem is used above. The functions concerned are only those which map in a one-to-one and conformal way the interior of the unit circle on the interior of a simple polygon. 
Thus the length of any level line of $\Sigma$ is not greater than the length of the boundary of $\Sigma$. The theorem of Hardy then implies that the length of any level line of $\Sigma$ is less than the length of the boundary of $\Sigma$.

That any sequence of level lines $\left\{G_{t_{n}}\right\}, t_{n}<t_{n+1}, n=1,2$, $3, \cdots$, and $\lim _{n \rightarrow \infty} t_{n}=1$, which belong to any given pencil of level lines $\left[G_{t}\right], 0<t<1$, of level lines of any planar region $\Sigma$ whose boundary is connected and contains more than one point is an approximating sequence of curves of $\Sigma$ follows easily from the one-to-one conformal mapping of the interior of the unit circle on $\Sigma$, which determines the sequence of level lines $\left\{G_{t_{n}}\right\}, t_{n}<t_{n+1}, n=1,2,3, \cdots$, as the image of a sequence of circles, $\left\{H_{n}\right\}, n=1,2,3, \cdots$, which are concentric with and interior to the unit circle and such that $\lim _{n \rightarrow \infty} r_{n}=1$, where $r_{n}$ is the length of the radius of the circle $H_{n}$. Evidently, the level line $G_{t_{n}}$ is in the interior of the level line $G_{t_{n+1}}$.

Now, if the boundary of $\Sigma$ is a rectifiable simple closed curve of length $l$, it follows readily from certain known results* that if $l_{n}$ is the length of the rectifiable curve $C_{n}$ of the approximating sequence of curves $\left\{C_{n}\right\}, n=1,2$, $3, \cdots$, of the region $\Sigma$ and if $\lim _{n \rightarrow \infty} l_{n}$ exists, then we have $\lim _{n \rightarrow \infty} l_{n} \geqq l$. Hence, with what has preceded, if $l_{t_{n}}$ is the length of the level line $G_{t_{n}}$ of the sequence of level lines $\left\{G_{t_{n}}\right\}$, $t_{n}<t_{n+1}, n=1,2,3, \cdots, \lim _{n \rightarrow \infty} t_{n}=1$, then $l_{t_{n}}<l$ and, since by the theorem of Hardy $l_{t_{n}}<l_{t_{n+1}}, n=1,2,3, \cdots$, it follows that $\lim _{n \rightarrow \infty} l_{t_{n}}=l$. Thus $\lim _{t \rightarrow 1} \Lambda(t)=l$ and the sequence of level lines $\left\{G_{t_{n}}\right\}, t_{n}<t_{n+1}, n=1,2,3, \cdots, \lim _{n \rightarrow \infty} t_{n}=1$, is an approximating sequence of curves of $\Sigma$ as specified in the second theorem.

Columbia University

* In particular, Theorem V, p. 519 of Hahn, Theorie der reellen Funktionen, vol. I. 